\title{
RANDOM MATRIX THEORY OF SCATTERING IN CHAOTIC AND DISORDERED MEDIA
}

\author{
Jean-Louis Pichard \\ Service de Physique de l'Etat Condensé, \\ CEA-Saclay, 91191 Gif sur Yvette cedex, France \\ pichard@drecam.saclay.cea.fr
}

Published in: Waves and Imaging through Complex Media ed. by P. Sebbah, Kluwer Academic Publishers (2001).

\begin{abstract}
We review the random matrix theory describing elastic scattering through zero-dimensional ballistic cavities (having chaotic classical dynamics) and quasi-one dimensional disordered systems. In zero dimension, general symmetry considerations (flux conservation and time reversal symmetry) are only considered, while the combination law of scatterers put in series is taken into account in quasi-one dimension. Originally developed for calculating the distribution of the electrical conductance of mesoscopic systems, this theory naturally reveals the universal behaviors characterizing elastic scattering of various scalar waves.
\end{abstract}

Keywords: Random Matrix Theory, Scattering Theory, Quantum Chaos, Anderson Localization, Disordered Systems.

This chapter is a short introductory review of the random matrix descriptions of elastic scattering. Additional informations can be found in more exhaustive reviews $[1,2,3]$. The more recent reviews are given by Bohigas [4], by Beenakker [5] and by Guhr, Müller-Groeling and Weidenmüller [6]. The basic references for random matrix theory are the book of Mehta [7] (see also Porter [8]) and the series of papers published by Dyson [9] in 1962.

\section{GAUSSIAN ENSEMBLES OF HERMITIAN MATRICES}

For a statistical description of a matrix ensemble, one has to define a measure of the space of the matrices having the required symmetries. If 
one is interested by the distribution of a restricted set of parameters suitable for describing the matrices (e. g. the eigenvalues of an Hermitian matrix), one has to use the system of coordinates using those parameters. The $\mathrm{J}$ acobian of the transformation (e. g. from matrix elements coordinates towards eigenvalue-eigenvector coordinates) yields correlations between those parameters. Those correlations (level repulsions) are at the origin of universal behaviors first observed in complex nuclei, then in small metallic particles, quantum billiards, hydrogen atom in a magnetic field, mesoscopic quantum systems, electro-magnetic cavities... The simplest illustration is given by the Gaussian ensembles of Hermitian matrices introduced in this section. Another illustration is given in the following section: the distribution of the radial parameters characterizing a scattering matrix $S$ or a transfer matrix $M$.

For doing statistics with real numbers, one defines the probability $P(d x)$ to have a real number $x$ inside an infinitesimal interval of length $d x: P(d x)=\rho(x) \mu(d x)$ where $\rho(x)$ is a density and $\mu(d x)=d x$ the measure of an infinitesimal interval of the real axis. Similarly, for doing statistics with matrices $X$, one defines the measure $\mu(d X)$ of an infinitesimal volume element $d X$ of the matrix space in which $X$ is defined and one gives a density probability $\rho(X)$. The measure $\mu(d X)$ is given by the symmetries of $X$, while the density $\rho(X)$ may contain physical assumptions (e.g. minimum information density given a few physical constraints).

For instance, let us introduce the Gaussian Orthogonal Ensemble (GOE) of real symmetric matrices $H$ and the probability distribution of their eigenvalues $E_{i}$. A real symmetric matrix $H=H^{T}=H^{*}$ of size $N$ has $\left(N^{2}+N\right) / 2$ independent entries. The infinitesimal volume element $d H$ has a measure $\mu(d H)$ given by the product of the infinitesimal variations $d H_{i j}$ of the $N(N+1) / 2$ independent entries:

$$
\mu(d H)=\prod_{i \leq j}^{N} d H_{i j} .
$$

A possible definition of the GOE density probability $\rho(H)$ is given by a maximum entropy criterion. Minimizing [10] the information entropy

$$
I(\rho(H))=-\int \rho(H) \ln \rho(H) \mu(d H)
$$

with an imposed expectation value for the trace of $H^{2}$ gives

$$
\rho(H) \propto \exp \left(-\frac{t r H^{2}}{2 \sigma^{2}}\right) .
$$


Since

$$
\exp \left(-\frac{t r H^{2}}{2 \sigma^{2}}\right) \mu(d H)=\prod_{i=1}^{N} \exp \left(-\frac{H_{i i}^{2}}{2 \sigma^{2}}\right) d H_{i i} \prod_{i<j}^{N} \exp \left(-\frac{H_{i j}^{2}}{\sigma^{2}}\right) d H_{i j},
$$

the $N(N+1) / 2$ independent matrix elements $H_{i j}(i \leq j)$ are uncorrelated variables with Gaussian distributions of variance $\sigma^{2}$ and $\sigma^{2} / 2$ for the diagonal and the off-diagonal entries respectively. This ratio between the variances is important since it makes the GOE ensemble invariant under change of basis: $\rho(H)$ is only function of the $N$ eigenvalues $E_{i}$ of $H$ through $\sum_{i=1}^{N} E_{i}^{2}$ and does not depend on the eigenvectors of $H$. To calculate $P\left(E_{1}, \ldots, E_{N}\right)$, one has to go from the parameterization of $H$ in terms of its matrix elements $H_{i j}$ to the parameterization of $H$ in terms of its eigenvalue-eigenvector coordinates. The Jacobian of this change of coordinates is at the basis of the level repulsion and of the spectral rigidity characteristic of usual random matrix theories.

A real symmetric matrix is diagonalizable by an orthogonal transformation. Let us define the measure of an orthogonal transformation $O_{N}$. We first consider a $2 d$ rotation $\mathrm{O}_{2}$ of angle $\theta$. One has

$$
O_{2}=\left(\begin{array}{cc}
\cos \theta & \sin \theta \\
-\sin \theta & \cos \theta
\end{array}\right)
$$

and by differentiation

$$
d O_{2}=O_{2}\left(\begin{array}{cc}
0 & d \theta \\
-d \theta & 0
\end{array}\right)
$$

Clearly, $\mu\left(d \mathrm{O}_{2}\right)=d \theta$ is the appropriate measure for the transformation $\mathrm{O}_{2}$. The generalization to an arbitrary $N \times N$ orthogonal transformation $O_{N}$ is straightforward:

$O_{N}^{T} O_{N}=I_{N} \quad\left(O_{N}+d O_{N}\right)^{T}\left(O_{N}+d O_{N}\right)=I_{N}$ $d O_{N}=O_{N} d A_{N} \quad d A_{N}^{T}=-d A_{N}$

where $I_{N}$ denotes the unit $N \times N$ matrix. $d A_{N}$ is a $N \times N$ real antisymmetric matrix and $\mu\left(d O_{N}\right)=\prod_{i<j}^{N} d A_{i j}$.

$H$ is diagonalizable by an orthogonal transformation $O: H=O H_{D} O^{T}$ where $H_{D}$ is a real diagonal matrix of entries $E_{i}$ and of measure $\mu\left(d H_{D}\right)=$ $\prod_{i=1}^{N} d E_{i}$. By differentiation, one gets

$$
\begin{array}{r}
d H=O d \mathcal{H} O^{T} \\
d \mathcal{H}=d A H_{D}-H_{D} d A+d H_{D}
\end{array}
$$

where we have used $d O=O d A$ and $d A^{T}=-d A$. The real symmetric matrix $d H$ is related to $d \mathcal{H}$ by an orthogonal transformation. The 
Jacobian is equal to one and $\mu(d H)=\prod_{i \leq j}^{N} d H_{i j}=\prod_{i \leq j}^{N} d \mathcal{H}_{i j}$. The product of the infinitesimal diagonal elements of $d \mathcal{H}$ gives $\mu\left(d H_{D}\right)$, the off-diagonal contribution gives $\prod_{i<j}^{N}\left|E_{i}-E_{j}\right| d A_{i j}$, and one eventually obtains the measure $\mu(d H)$ in terms of the measures $\mu(d O)$ and $\mu\left(d H_{D}\right)$

$$
\mu(d H)=\prod_{i<j}^{N}\left|E_{i}-E_{j}\right| \mu\left(d H_{D}\right) \mu(d O) .
$$

In terms of the eigenvalue-eigenvector coordinates of $H$, the GOE distribution becomes:

$$
P(d H) \mu(d H)=P\left(E_{1}, \ldots, E_{N}\right) \mu\left(d H_{D}\right) \mu(d O)
$$

where the joint probability distribution of the eigenvalues is identical to the Gibbs factor of a set of $N$ point charges free to move on the real axis of the complex plane with a pairwise logarithmic repulsion and a quadratic confining potential at an inverse temperature $\beta=1$ :

$$
P\left(E_{1}, \ldots, E_{N}\right) \propto \exp \left[-\beta \sum_{i<j}^{N} \ln \left|E_{i}-E_{j}\right|+\sum_{i=1}^{N} \frac{E_{i}^{2}}{2 \sigma^{2}}\right]
$$

The pairwise repulsion coming from the Jacobian makes unlikely level degeneracies and explains why dramatically non-random an energy-level series really is. This is the Coulomb gas analogy usual in Random Matrix Theory. To appreciate how this random matrix approach is adapted to include symmetry breaking effects, let us assume that the matrix $H$ is the Hamiltonian of an electron moving in a chaotic cavity. Applying a magnetic field removes time reversal symmetry, $H$ becomes hermitian $\left(H=H^{\dagger}\right)$ and $\mu(d H)=\prod_{i=1}^{N} d H_{i i}^{1} \prod_{i<j} d H_{i j}^{1} d H_{i j}^{2}$, taking into account the infinitesimal variations of the real and imaginary parts of its matrix elements $\left(H_{i j}=H_{i j}^{1}+i H_{i j}^{2}\right)$. $H$ is now diagonalizable by a unitary transformation $U$ and $d U=U d a$ where $d a$ is an infinitesimal anti-hermitian matrix $\left(d a=-d a^{\dagger}\right)$, and $\mu(d U)=\prod_{i=1}^{N} d a_{i i}^{2} \prod_{i<j}^{N} d a_{i j}^{1} d a_{i j}^{2}$. One obtains for hermitian matrices $d H=U d \mathcal{H} U^{\dagger}$ where $d \mathcal{H}=d a H_{D}+d H_{D}-H_{D} d a$. The Jacobian of a unitary transformation being equal to one, one eventually finds $\mu(d H)=\prod_{i<j}\left|E_{i}-E_{j}\right|^{2} \mu\left(d H_{D}\right) \mu(d U) /\left(\prod_{i} d a_{i i}^{2}\right)$, the square coming from the fact that the non diagonal contribution of $d \mathcal{H}$ is now complex. Breaking time reversal symmetry, one keeps the Coulomb gas analogy, with a temperature divided by a factor two $(\beta=1 \rightarrow 2)$. For electrons of spin $1 / 2$, one can also break spin rotation symmetry (SRS) by spin orbit scattering, an effect which preserves time reversal symmetry (TRS). The matrix elements of $H$ are no longer real $(\beta=1)$ or 
complex $(\beta=2)$, but quaternion real $(\beta=4)$ and the level distribution is still given by the Coulomb gas analogy with a temperature divided by a factor $4: \beta=1 \rightarrow 4$.

The main feature of those three Gaussian ensembles of random matrices is that $\rho(H)$ does not couple eigenvalue and eigenvector variables. These ensembles are invariant under canonical transformations: orthogonal transformations $(\beta=1)$ when the system invariant under TRS and SRS symmetries, unitary transformations $(\beta=2)$ in the absence of TRS and symplectic transformations $(\beta=4)$ with TRS and without SRS. The eigenvectors are totally random, the measure of the matrices $O$ or $U$ are given by the Haar measures over the orthogonal or unitary groups respectively, and the integration over the eigenvectors is trivial. This is the totally random character of the eigenvectors which makes the energy levels correlated (pairwise logarithmic repulsion) and subject to universal symmetry breaking effects (e.g. $\beta=1,4 \rightarrow 2$ when TRS is broken).

\section{RADIAL PARAMETERIZATION OF SCATTERING MATRICES $S$ AND MEASURES}

Similar random matrix theories can be adapted to matrices having different symmetries and can give the joint probability distribution of a subset of variables which can be used for their parameterization. Another example is provided by the unitary matrices describing complex elastic scatterers. Let us consider a perfect waveguide characterized by $N$ quantized modes propagating to the right and $N$ time reversed modes propagating to the left. Let us introduce in the middle of this wave guide a complex elastic scatterer described by its $2 N \times 2 N$ scattering matrix $S$. This matrix describes the various transmission and reflection amplitudes to the right or to the left, has to be unitary for conserving the flux amplitudes $\left(S S^{\dagger}=S^{\dagger} S=I_{2 N}\right)$, and must be symmetric $\left(S=S^{T}\right)$ if one has time reversal invariant scattering:

$$
S=\left(\begin{array}{cc}
r & t^{\prime} \\
t & r^{\prime}
\end{array}\right)
$$

The $N \times N$ matrices $t$ and $t^{\prime}$ describe transmission amplitudes of the incoming fluxes to the right and left directions respectively, while $r$ and $r^{\prime}$ describe reflections. Let us consider time reversal invariant scattering where $S$ is symmetric and can be decomposed as

$$
\begin{array}{r}
S=U^{T} \cdot U \\
S+d S=U^{T}\left(I_{2 N}+i d M\right) U
\end{array}
$$


where $U$ and $d M$ are respectively a unitary and an infinitesimal real symmetric matrices. This decomposition is not unique, one can multiply $U$ by an arbitrary orthogonal transformation $O(U \rightarrow U O)$ but the measure $\mu(d S)=\prod_{i \leq j} d M_{i j}$ is uniquely defined since the Jacobian of the transformation $d M \rightarrow d \mathcal{M}=O d M O^{T}$ is equal to one. $\mu(d S)$ was expressed by Dyson [9] in terms of the eigenvectors and eigenvalues of $S$ and their associated measures. The original motivation in Dyson's work was not at all to study a scattering problem, but to use the eigenvalue distribution of $S$ for describing energy-level statistics (the eigenvalues of $S$ being confined on the unit circle of the complex plane, one does not need the somewhat artificial GOE quadratic confining potential). For a scattering problem, the eigenvalue-eigenvector parameterization of $S$ is not adapted and we introduce a more convenient one using 2 unitary $N \times N$ matrices $u_{1}$ and $u_{2}$ and a diagonal $N \times N$ matrix $\Lambda$ with $N$ real positive diagonal entries $\lambda_{1}, \ldots, \lambda_{N}$. Denoting $T=(1+\Lambda)^{-1}$ and $R=\Lambda(1+\Lambda)^{-1}, S$ can be written as:

$$
S=\left(\begin{array}{cc}
u_{1} & 0 \\
0 & u_{2}
\end{array}\right)\left(\begin{array}{cc}
-\sqrt{R} & \sqrt{T} \\
\sqrt{T} & \sqrt{R}
\end{array}\right)\left(\begin{array}{cc}
u_{1}^{T} & 0 \\
0 & u_{2}^{T}
\end{array}\right)
$$

In this parameterization, the transmission and reflection matrices become

$$
\begin{aligned}
t=u_{2} \sqrt{T} u_{1}^{T} & t^{\prime}=u_{1} \sqrt{T} u_{2}^{T} \\
r=-u_{1} \sqrt{R} u_{1}^{T} & r^{\prime}=u_{2} \sqrt{R} u_{2}^{T}
\end{aligned}
$$

and $T$ and $R$ contain the eigenvalues of $t t^{\dagger}$ and $r r^{\dagger}$ (transmission and reflection eigenvalues). One can note that the transfer matrix $M$ which gives the flux amplitudes of the right side of the scatterer in terms of the flux amplitudes of its left side can be written using the same parameterization as

$$
M=\left(\begin{array}{cc}
u_{2} & 0 \\
0 & u_{2}^{*}
\end{array}\right)\left(\begin{array}{cc}
\sqrt{I_{N}+\Lambda} & \sqrt{\Lambda} \\
\sqrt{\Lambda} & \sqrt{I_{N}+\Lambda}
\end{array}\right)\left(\begin{array}{cc}
u_{1}^{T} & 0 \\
0 & u_{1}^{\dagger}
\end{array}\right)
$$

$M$ is pseudo unitary (flux conservation)

$$
M\left(\begin{array}{cc}
I_{N} & 0 \\
0 & -I_{N}
\end{array}\right) M^{\dagger}=M^{\dagger}\left(\begin{array}{cc}
I_{N} & 0 \\
0 & -I_{N}
\end{array}\right) M=\left(\begin{array}{cc}
I_{N} & 0 \\
0 & -I_{N}
\end{array}\right)
$$

and when one has TRS $(\beta=1), M$ must also satisfy the requirement:

$$
M^{*}=\left(\begin{array}{cc}
0 & I_{N} \\
I_{N} & 0
\end{array}\right) M\left(\begin{array}{cc}
0 & I_{N} \\
I_{N} & 0
\end{array}\right)
$$


$M$ has the advantage to be multiplicative when one puts the scatterers in series. For the derivation of this parameterization of $S$, see Ref.[11], and use the relation between the matrices $M$ and $S$. To understand the interest of this parameterization, we introduce the variables $x_{i}$ from $\lambda_{i}=\sinh ^{2} x_{i}$. One can see than $M$ is decomposed in the product of a unitary transformation, followed by $N$ hyperbolic rotations of angle $x_{i}$ :

$$
\left(\begin{array}{cc}
\sqrt{1+\lambda_{i}} & \sqrt{\lambda_{i}} \\
\sqrt{\lambda}_{i} & \sqrt{1+\lambda_{i}}
\end{array}\right) \rightarrow\left(\begin{array}{cc}
\cosh x_{i} & \sinh x_{i} \\
\sinh x_{i} & \cosh x_{i}
\end{array}\right)
$$

before a second unitary transformation. The $N$ parameters $\lambda_{i}$ are called the radial parameters of $M$ (or $S$ ).

Diagonalizing the matrix containing those parameters in the new parameterization of $S$ by the orthogonal matrix $O$

$$
O=\frac{1}{\sqrt{2}}\left(\begin{array}{cc}
\left(I_{N}-\sqrt{R}\right)^{1 / 2} & \left(I_{N}+\sqrt{R}\right)^{1 / 2} \\
\left(I_{N}-\sqrt{R}\right)^{1 / 2} & -\left(I_{N}+\sqrt{R}\right)^{1 / 2}
\end{array}\right)
$$

and denoting

$$
U=\left(\begin{array}{cc}
u_{1} & 0 \\
0 & u_{2}
\end{array}\right)
$$

and

$$
I=\left(\begin{array}{cc}
i I_{N} & 0 \\
0 & -i I_{N}
\end{array}\right)
$$

one can write $S=Y Y^{T}$ where the unitary matrix $Y=U O I$. Defining the infinitesimal anti-hermitian and real antisymmetric matrices $d A$ and $d B$ from $d U=U d A$ and $d O=O d B$, using $d S=i Y d M Y^{T}$, one obtains:

$$
\begin{aligned}
i d M & =d C+d C^{T} \\
d C & =I^{*}\left(d B+O^{T} d A O\right) I
\end{aligned}
$$

which allows us to write the Jacobian matrix of the change of coordinates $d M_{i j} \rightarrow\left(d A_{i j}, d B_{i j}\right)$. This matrix has a simple block diagonal form and its determinant gives the measure $\mu(d S)$ in terms of the measures $\mu(d \Lambda)=\prod_{i=1}^{N} d \lambda_{i}$ and $\mu(d U)=\prod_{i=1}^{2} \mu\left(d u_{i}\right)$. We have sketched the derivation when $S$ is unitary symmetric $(\beta=1)$, but the extension to the three possible symmetry classes is straightforward and gives for $\mu_{\beta}(d S)$ the general form [12]

$$
\begin{array}{r}
\mu_{\beta}(d S)=P_{\beta}\left(\lambda_{1}, \ldots, \lambda_{N}\right) \mu(d \Lambda) \mu(d U) \\
P_{\beta}\left(\lambda_{1}, \ldots, \lambda_{N}\right)=\exp -\beta H_{\beta}\left(\lambda_{1}, \ldots, \lambda_{N}\right)
\end{array}
$$




$$
\begin{array}{r}
H_{\beta}\left(\lambda_{1}, \ldots, \lambda_{N}\right)=-\sum_{i<j}^{N} \ln \left|\lambda_{i}-\lambda_{j}\right|+\sum_{i=1}^{N} V_{\beta}\left(\lambda_{i}\right) \\
V_{\beta}(\lambda)=\left(N+\frac{2-\beta}{2 \beta}\right) \ln (1+\lambda)
\end{array}
$$

If the scattering is not time reversal symmetric $(\beta=2), S$ is no longer symmetric and one needs two additional unitary matrices $u_{3}$ and $u_{4}$ for parameterizing $S$ and $\mu(d U)=\prod_{i=1}^{4} \mu\left(d u_{i}\right)$. If one considers scattering of spin $1 / 2$ particles by a TRS scatterer which removes SRS (spin-orbit scattering), $\beta=4$.

One can similarly show that the measure for the transfer matrices [2] is given in terms of the radial parameters $\lambda_{i}$ by:

$$
\mu_{\beta}(d M)=\prod_{i<j}^{N}\left|\lambda_{i}-\lambda_{j}\right|^{\beta} \mu(d \Lambda) \prod_{i=1}^{2,4} \mu\left(d u_{i}\right)
$$

\section{ZERO DIMENSIONAL CHAOTIC SCATTERING}

Let us assume that the scatterer represented by the matrix $S$ is a ballistic cavity of irregular shape having chaotic classical dynamics, as sketched in Fig. 1. We have in mind long scattering trajectories corre-

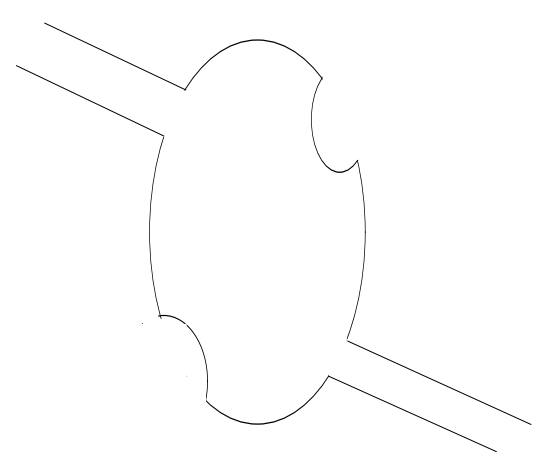

Figure 1. Scheme of a cavity giving rise to zero-dimensional chaotic scaterring.

sponding to particles reflected many times inside the cavity before being transmitted or reflected. Let us assume that the shape of the cavity is 
slightly modified, or that the wave length of the incoming fluxes varies. The scattering will be deeply re-organized and a statistical description of the fluctuations of the scattering amplitudes becomes necessary. To this end, we need to define a statistical ensemble of scattering matrices $S$ and we will assume that the scatterer will visit this ensemble when one varies a tunable parameter (shape of the cavity, wave-length, applied magnetic field reorganizing the quantum interferences if we consider electron elastic scattering). The simplest ensemble is the one where all the scattering processes are equiprobable, which does not contain any information about the system excepted its basic symmetries. Those ensembles of minimum information entropy for $S$ are the circular ensembles [9] introduced by Dyson in 1962, for which the probability to find $S$ inside a volume element $d S$ is

$$
P(d S)=\frac{1}{V_{\beta}} \mu_{\beta}(d S)
$$

where $V_{\beta}$ is a normalization constant. One obtains for the radial parameters $\lambda_{i}$ a Coulomb gas analogy very similar to the GOE-GUE-GSE Coulomb gas analogies for the energy level of a random Hamiltonian, excepted two noticeable differences: (i) the $\lambda_{i}$ are real positive in contrast to the $E_{i}$ which are only real, the Coulomb gas is free to move only on the positive part of the real axis in the complex plane (ii) the confining potential $V_{\beta}(\lambda)$ is implied by the symmetries of $S$ (in contrast to the quadratic potential given by a certain choice of $\rho(H)$ ) and depends on the symmetry parameter $\beta$.

Let us see the implication for the total transmission probability $\mathcal{T}=$ $t r t t^{\dagger}=\sum_{i=1}^{N} T_{i}=\sum_{i=1}^{N}\left(1+\lambda_{i}\right)^{-1}$. When $S$ gives Fermi wave scattering by a mesoscopic scatterer coupling to electron reservoirs, $\mathcal{T}$ gives its electrical conductance in units of $2 e^{2} / h$ (Landauer formula).

If $N=1$ (single mode wave guide), the probability distribution of $\mathcal{T}$ exhibits strong symmetry breaking effects (see Fig. 2):

$$
P(\mathcal{T})=\frac{\beta}{2} \mathcal{T}^{\beta / 2-1}
$$

If one measures the electrical conductance of a chaotic cavity (quantum billiard) coupled to two electron reservoirs by single mode leads, the conductance is likely to be close to 0 if one has TRS and SRS, to have a value uniformly distributed between 0 and $2 e^{2} / h$ if one applies a small magnetic field (no TRS), and a value close to $2 e^{2} / h$ if the electron reflection on the walls of the cavity is accompanied by spin-orbit scattering (no SRS). The distribution $P(\mathcal{T})$ can be explored by changing the Fermi energy with a metallic gate, by small deformations of the cavity or by applying a magnetic field. 


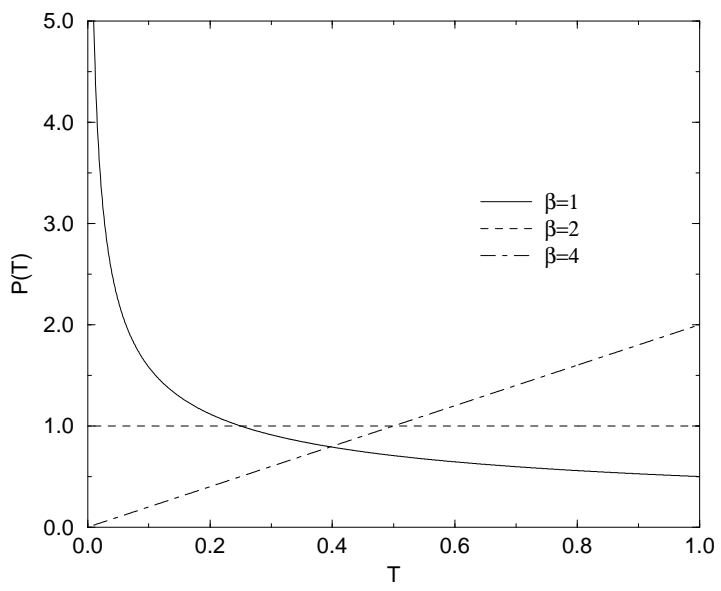

Figure 2. Symmetry breaking effect on the distribution $P(\mathcal{T})$ for a chaotic cavity coupled to leads via single mode contacts $(N=1)$.

If $N>>1$ the symmetry breaking effects are much smaller. A first one is the suppression, when there is no TRS, of a small "weak localization" correction to the average transmission $\langle\mathcal{T}\rangle$, the second effect of removing TRS is to halve the universal variance of $\mathcal{T}$.

\subsection{WEAK LOCALIZATION CORRECTIONS}

The large $N$ limit of the density $\rho(\lambda)$

$$
\rho_{\beta}(\lambda)=\sum_{i=1}^{N} \int_{R^{+}} \ldots \int_{R^{+}} \prod_{i=1}^{N} d \lambda_{i} P_{\beta}\left(\lambda_{1}, \ldots, \lambda_{N}\right) \delta\left(\lambda-\lambda_{i}\right)
$$

can be calculated using an equation derived by Dyson [13]

$$
\int_{R^{+}} \frac{\rho_{\beta}\left(\lambda^{\prime}\right) d \lambda^{\prime}}{\lambda-\lambda^{\prime}}+\frac{\beta-2}{2 \beta} \frac{d \ln \rho_{\beta}(\lambda)}{d \lambda}=\frac{d V_{\beta}(\lambda)}{d \lambda}
$$

Taking the $V_{\beta}(\lambda)$ characterizing the three circular ensembles for $S$, one gets for the ensemble averaged total transmission:

$$
<\mathcal{T}>=\int_{R^{+}} \frac{\rho_{\beta}(\lambda)}{1+\lambda}=\frac{N}{2}+\frac{\beta-2}{4 \beta}+O\left(\frac{1}{N}\right)
$$

The term $\propto N$ is obvious: having chaotic scattering, the probability to be reflected equals the probability to be transmitted. However there is a small correction of order 1 which reduces by $-1 / 4 \mathcal{T}$ when there is 
TRS and SRS, which disappears without TRS and which enhances $\mathcal{T}$ by a factor $1 / 8$ without SRS. This is the analog in a quantum billiard of the well-known weak-localization corrections to the Boltzmann-Drude conductance of a disordered system.

\subsection{UNIVERSAL CONDUCTANCE FLUCTUATIONS}

One of the main phenomena which is naturally explained by random matrix theory [14] are the "universal conductance fluctuations" (UCF) characterizing mesoscopic conductors. When one varies the "conductance" $\mathcal{T}$ with an external parameter (Fermi wave length, magnetic field ...) one generates fluctuations of magnitude independent of $\langle\mathcal{T}\rangle$. To calculate $\left\langle\delta \mathcal{T}^{2}>\right.$ one needs to know the density-density correlation function of the $\lambda$-parameters. In the limit $N \rightarrow \infty$, one can simply calculate [15] this variance. Exploiting the Coulomb gas analogy, one can write

$$
K_{\beta}\left(\lambda, \lambda^{\prime}\right)=<\sum_{i j} \delta\left(\lambda-\lambda_{i}\right) \delta\left(\lambda-\lambda_{j}\right)>-\rho_{\beta}(\lambda) \rho_{\beta}\left(\lambda^{\prime}\right)
$$

as a functional derivative:

$$
K_{\beta}\left(\lambda, \lambda^{\prime}\right)=-\frac{1}{\beta} \frac{\partial \rho_{\beta}(\lambda)}{\partial V_{\beta}\left(\lambda^{\prime}\right)}
$$

When $N \rightarrow \infty, V_{\beta}(\lambda) \rightarrow \int_{R^{+}} \ln \left|\lambda-\lambda^{\prime}\right| \rho_{\beta}(\lambda)+$ const (this amounts to neglect the term responsible for the weak localization correction in Eq. (33). In the large $N$-limit, $V_{\beta}(\lambda)$ becomes a linear functional of $\rho\left(\lambda^{\prime}\right)$. This implies an important result: $K_{\beta}\left(\lambda, \lambda^{\prime}\right)$ depends only on the nature of the pairwise repulsion between the radial parameters and becomes independent of the confining potential $V_{\beta}(\lambda)$. The evaluation of the functional derivative giving $K_{\beta}\left(\lambda, \lambda^{\prime}\right)$ is straightforward:

$$
\lim _{N \rightarrow \infty} K_{\beta}\left(\lambda, \lambda^{\prime}\right)=-\frac{1}{\pi^{2} \beta} \ln \frac{\sqrt{\lambda}-\sqrt{\lambda}^{\prime}}{\sqrt{\lambda}+\sqrt{\lambda}^{\prime}} .
$$

to eventually give

$$
<\delta^{2}(\mathcal{A})>\rightarrow \frac{-1}{\beta \pi^{2}} \int_{R^{+}} \int_{R+} d \lambda d \lambda^{\prime} \ln \frac{\sqrt{\lambda}-\sqrt{\lambda}^{\prime}}{\sqrt{\lambda}+\sqrt{\lambda}^{\prime}} \frac{d a(\lambda)}{d \lambda} \frac{d a\left(\lambda^{\prime}\right)}{d \lambda^{\prime}}
$$

for the variance $\left\langle\delta^{2} A>\right.$ of a linear statistics $A=\sum_{i=1}^{N} a\left(\lambda_{i}\right)$ of the $\lambda$-parameter. Taking $a(\lambda)=(1+\lambda)^{-1}$, one gets

$$
<\delta^{2} \mathcal{T}>=\frac{2}{16 \beta}
$$


The variance of $\mathcal{T}$ is just a number which depends on $\beta$ but not on $<\mathcal{T}>$.

When $N$ is finite, weak localization corrections and variances can be exactly calculated using a method introduced by Mehta, Gaudin and Dyson. Using a set of orthogonal polynomials $(\beta=2)$ or sheworthogonal polynomials $(\beta=1,4)$, one can perform the integration of $P_{\beta}\left(\lambda_{1}, \ldots, \lambda_{N}\right)$ over an arbitrary set of $\lambda$-parameters in order to have $\rho_{\beta}(\lambda), K_{\beta}\left(\lambda, \lambda^{\prime}\right)$ and higher order correlation functions. Going to the variables $T_{i}=\left(1+\lambda_{i}\right)$ the polynomials required [12] to perform the integrals for $\beta=2$ are the Legendre polynomials.

\section{MANY CHANNEL DISORDERED WAVE GUIDE}

So far, we have done the simplest random matrix exercise where all the scatterers are taken with a uniform distribution. Those circular ensembles are suitable to describe scattering in zero-dimensional chaotic cavities, as it has been numerically checked. Another exactly solvable case is provided by a $1 d$ series of scatterers. Let us consider a quasi- $1 d$ disordered wire (or wave guide) with $N$ channels, as sketched in Fig. 3.

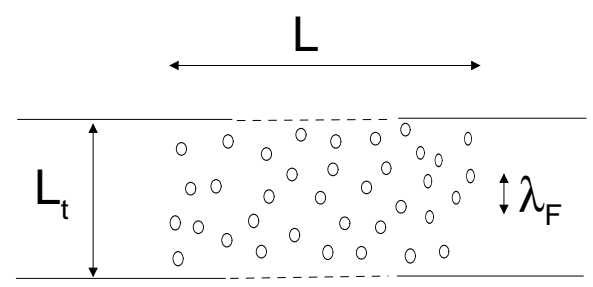

Figure 3. Scheme of a quasi-1d disoredered wave guide of length $L>>L_{t}$ with $N=\left(L_{t} / \lambda_{F}\right)^{d-1}$ modes.

Typically, if the (Fermi) wavelength is $\lambda_{F}$, and $L_{t}^{d-1}$ the transverse section, one has $N=\left(L_{t} / \lambda_{F}\right)^{d-1}$. Let us consider a "building block" of length $\delta L$ and of transfer matrix $M_{\delta L}$. A suitable statistical ensemble for 
$M_{\delta L}$ is given by a maximum entropy ensemble where $\langle\mathcal{T}\rangle$ is imposed. If $\delta L$ is larger than the elastic mean free path $l$, a natural requirement is to impose Ohm's law (the conductance $<g>\propto<\mathcal{T}>\propto N l(\delta L)^{-1}$ ). This gives for the building block $P\left(M_{\delta L}\right) \propto \exp -(A<\mathcal{T}>) \mu(d M)$, where $A$ is the Lagrange multiplier associated to the imposed constraint. This ensemble preserves the logarithmic pairwise interaction between the $\lambda$-parameter and the distribution of the auxiliary unitary matrices $u_{i}$ derived for the circular ensembles, but gives rise to a different potential $V_{\beta}(\lambda)$. Considering a $1 d$ series of such building blocks, of length $L / l$, and exploiting the multiplicative combination law of $M$ $\left(M_{L+\delta L}=M_{L} \cdot M_{\delta L}\right)$, one can derive a Fokker-Planck equation [5, 16, 17] for $P_{\beta}\left(\lambda_{1}, \ldots, \lambda_{N}, L / l\right)$ :

$$
\begin{array}{r}
\frac{\partial P_{\beta}\left(\lambda_{1}, \ldots, \lambda_{N}\right)}{\partial L}=D \Delta_{\lambda} P_{\beta}\left(\lambda_{1}, \ldots, \lambda_{N}\right) \\
D=\frac{2}{(\beta N+2-\beta) l} \\
\Delta_{\lambda}=\sum_{i=1}^{N} \frac{\partial}{\partial \lambda_{i}} \lambda_{i}\left(1+\lambda_{i}\right) J \frac{\partial}{\partial \lambda_{i}} \frac{1}{J} \\
J=\prod_{i<j}^{N}\left|\lambda_{i}-\lambda_{j}\right|^{\beta}
\end{array}
$$

In this statistical description of $M$ (or $S$ ), the radial parameters and the matrices $u_{i}$ are statistically uncorrelated. The $u_{i}$ remain distributed with Haar measure over the $N \times N$ unitary group. Increasing the number $L / l$ of blocks put in series only changes the statistics of the radial parameters as given by the above Fokker-Planck equation, This is the limitation of this "isotropic" model which allows us to solve it entirely and to describe quasi- $1 d$ localization. But the transverse system dimensions appear only through the parameter $N$. A strip of purely $1 d$ transverse section and a bar with a $2 d$ section are treated the same way: within the $0 d$ approximation for the transverse dynamics. When $N=1, J=$ 1 and the resulting equation was originally derived [18] in 1959 in a work entitled: "waveguides with random inhomogeneities and Brownian motion in the Lobachevsky plane". The nature of the Fokker-Planck equation was indeed correctly identified as a "Heat equation in a space of negative curvature". Looking in the mathematical literature, one can realize that $\Delta_{\lambda}$ is the radial part of the Laplace-Beltrami operator in the space of the transfer matrices $M$. Increasing $L$ yields for $M$ an "isotropic" (invariant under the unitary transformation $u_{i}$ ) Brownian 
motion in the transfer matrix space which is only characterized by the diffusion constant $D$.

\subsection{QUASI-1D LOCALIZATION}

When $L$ increases and exceeds the localization length $\xi$, the system becomes an Anderson insulator and it is more convenient to use the variables $x_{i}=L / \xi_{i}\left(\lambda_{i}=\sinh ^{2} x_{i}\right)$, where the lengths $\xi_{i}$ characterize the exponential decays of the transmission channels of the quasi- $1 d$ scatterer $\left(T_{i} \approx \exp -\left(2 L / \xi_{i}\right)\right)$. When $L \rightarrow \infty$ the variables $1 / \xi_{i}$ are given by the Lyapounov exponents of the multiplicative transfer matrix $M$, giving $N$ decay lengths $\xi_{N}<<\xi_{N-1}<<\xi_{1}$, the largest of them defining [19] the localization length $\xi$ of the quasi-1d system. The Fokker-Planck equation becomes:

$$
\begin{array}{r}
\frac{\partial P_{\beta}\left(x_{1}, \ldots, x_{N}\right)}{\partial L}=D \sum_{i=1}^{N} \frac{\partial}{\partial x_{i}}\left(P_{\beta}+\beta P_{\beta} \frac{\partial \Omega}{\partial x_{i}}\right) \\
\Omega=\sum_{i<j}^{N} \ln \left|\sinh x_{i}^{2}-\sinh ^{2} x_{j}\right|-\frac{1}{\beta} \sum_{i=1}^{N} \ln \left|\sinh x_{i}\right|
\end{array}
$$

where the different channels are coupled via $\Omega$. However, when $L \rightarrow \infty$, $\left|\sinh ^{2} x_{i}-\sinh ^{2} x_{j}\right| \approx \exp 2 x_{i}$ when $x_{i}>>x_{j}$, the channels become decoupled and $\Omega \rightarrow-(2 / \beta) \sum_{i=1}^{N}(1+\beta N-\beta) x_{i}$. The Fokker-Planck equation becomes solvable and gives $[1]$ :

$$
P_{\beta}\left(x_{1}, \ldots, x_{N}, L\right)=\left(\frac{\gamma l}{2 \pi L}\right)^{\frac{N}{2}} \prod_{i=1}^{N} \exp \left(-\frac{\gamma l}{2 L}\left(x_{i}-\frac{L}{\xi_{i}}\right)^{2}\right)
$$

where $\xi_{i}=(\gamma l) /(1+\beta N-\beta)$ and $\gamma=\beta N+2-\beta$. One gets two important results:

(i) a universal symmetry breaking effect for the localization length $\xi$ when $\beta=1 \rightarrow 2$ :

$$
\xi=(\beta N+2-\beta) l
$$

(ii) a normal distribution for $\ln \mathcal{T}$ where $-2<\ln \mathcal{T}\rangle=\left\langle\delta^{2} \ln \mathcal{T}\right\rangle$. Those symmetry breaking effects have been observed in magneto-transport measurements performed in disordered wires $[20,21,22]$, where the conductance $g \propto \exp -(2 L / \xi)$ and $\xi=(N+1) l \rightarrow 2 N l$ when $\beta=1 \rightarrow 2$. However this theory neglecting electron-electron interactions, it would be important to check those universal symmetry breaking effects in the localized regime using other waves (light, sound ...). 


\subsection{MAPPING ONTO A CALOGERO-SUTHERLAND MODEL OF INTERACTING FERMIONS}

When $L \leq \xi$, the system is a disordered conductor where a number $N_{\text {eff }}<N$ of channels are still opened, giving $\langle\mathcal{T}\rangle \approx N l / L$ (Ohm's law) if one ignores the (small) weak-localization corrections. Approximations can be used also in this limit $(L<<\xi)$ to calculate the quasi-1d weak-localization corrections $\delta \mathcal{T}=(\beta-2) /(3 \beta)$ and the UCF variance $2 /(15 \beta)$. One can notice that those values valid for the quasi- $1 d$ wire are close, but not identical to those derived from the circular ensembles. The small difference between the UCF variances tells us (see the arguments given for having $K\left(\lambda, \lambda^{\prime}\right)$ in the large $N$-limit) that the radial parameters cannot have [15] exactly the pairwise logarithmic repulsion in quasi- $1 d$. This can be understood when $\beta=2$ where one can solve the Fokker-Planck equation for any values of $N$, using a transformation originally introduced by Sutherland to solve Dyson's Brownian motion model. The distribution $P_{\beta}\left(x_{1}, \ldots, x_{N}, L\right)$ is related $[5,23]$ to a wave function $\Psi\left(x_{1}, \ldots, x_{N}, L\right)$ by the transformation

$$
P=\Psi \exp -\left(\frac{\beta \Omega}{2}\right)
$$

and the Fokker-Planck equation for $P$ becomes a Schrödinger equation for $\Psi$ in imaginary time.

$$
-l \frac{\partial \Psi}{\partial L}=H \Psi
$$

where the Hamiltonian is given by

$$
\begin{array}{r}
H=\frac{-1}{2 \gamma} \sum_{i=1}^{N}\left(\frac{\partial^{2}}{\partial x_{i}^{2}}+\frac{1}{\sinh ^{2} 2 x_{i}}\right)+(\beta-2) U\left(x_{i}, x_{j}\right) \\
U\left(x_{i}, x_{j}\right)=\frac{\beta}{2 \gamma} \sum_{i<j}^{N} \frac{\sinh ^{2} 2 x_{j}+\sinh ^{2} 2 x_{i}}{\left(\cosh 2 x_{j}-\cosh ^{2} 2 x_{i}\right)^{2}}
\end{array}
$$

For $\beta=2$, the "particles" do not interact and the equation can be solved to give [23] a small change in the Coulomb gas analogy for the radial parameters:

$$
-\ln \left|\lambda_{i}-\lambda_{j}\right| \rightarrow-\frac{1}{2} \ln \left|\lambda_{i}-\lambda_{j}\right|-\frac{1}{2} \ln \left|\operatorname{arsinh}^{2} \sqrt{\lambda_{\mathrm{i}}}-\operatorname{arsinh}^{2} \sqrt{\lambda_{\mathrm{j}}}\right|
$$

yielding the change $\left\langle\delta^{2} \mathcal{T}\right\rangle=\frac{2}{16 \beta} \rightarrow \frac{2}{15 \beta}$. 
For $\beta \neq 2$, one has not yet found how to solve the Schrödinger equation. Let us note that the Calogero-Sutherland Hamiltonian derived from the original Dyson's Brownian motion model is now solved [24] when $\beta / 2=p / q$ where $p$ and $q$ are integer.

\section{SUMMARY}

We have seen how to derive the probability distributions of $S$ and how to extract the distribution of $\mathcal{T}$ using very little information (symmetries, combination law in quasild). This can be simply done as far as the radial parameters are decoupled from the auxiliary matrices $u_{i}$, which limits the method to $0 d$ and quasi- $1 d$. A more difficult task remaining to be achieved is to go beyond this limit, and to describe elastic scattering in $2 d$ and $3 d$, including possible Anderson localization. One can ask to what extend a real chaotic cavity or a quasi- $1 d$ disordered wave-guide is accurately described by those random matrix theories. Numerical calculations [25] of the distribution of $\mathcal{T}$ in (suitably) designed chaotic cavities (suitably) connected to two many-channel ballistic waveguides confirm the random matrix results. For quasi- $1 d$ disordered wires, a field theory approach has been derived [26] assuming local diffusive dynamics. One obtains a non-linear $\sigma$-model which gives using supersymmetry < $\mathcal{T}(\mathcal{L})>$ and $\left\langle\delta^{2} \mathcal{T}(L)>\right.$ in the large $N$-limit. The results turn out to coincide $[27,28]$ with those given by the Fokker-Planck equation in this limit. The finite $N$ behaviors which are calculable by the Fokker-Planck equation are still out of reach using the $\sigma$-model, as well as the moments of $\ln \mathcal{T}$ which are the statistically meaningful observables (related to a normal distribution) in the localized limit.

\section{Acknowledgments}

My own research on this random matrix theory adapted to scattering was published in a series of papers done with C.W.J. Beenakker, Y. Imry, R. A. Jalabert, P. A. Mello, K. Muttalib, K. M. Frahm, K. Slevin, A. D. Stone and N. Zanon, collaborations which are gratefully acknowledged.

\section{References}

[1] J.-L. Pichard, in Quantum coherence in Mesoscopic systems, edited by B. Kramer, NATO ASI Series B254 (plenum New York) p369 (1991).

[2] A. D. Stone, P. A. Mello, K. A. Muttalib and J.-L. Pichard, in Mesoscopic Phenomena in Solids. edited by B. L. Altshuler, P. A. Lee and R. A. Webb, (North Holland, Amsterdam) p 369 (1991).

[3] P. A. Mello, in Mesoscopic Quantum Physics, edited by E. Akkermans, G. Montambaux, J.-L. Pichard and J. Zinn-Justin, (North Holland, Amsterdam), p 435, 
(1995).

[4] O. Bohigas, in Chaos and Quantum Physics, edited by M.-J. Giannoni, A. Voros and J. Zinn-Justin, (North Holland, Amsterdam), p 87, (1990).

[5] C. W. J. Beenakker, Rev. Mod. Phys. 69, p731, (1997).

[6] T. Guhr, A. Müller-Groeling and H. A. Weidenmüller, Phys. Rep. 299, p 189, (1998).

[7] M. L. Mehta, Random Matrices (Academic, New-York), (1991).

[8] C. E. Porter, Statistical Theories of Spectra: Fluctuations, (Academic, New-York) (1965).

[9] F. J. Dyson, J. Math. Phys. 3, p140, 157, 1191 and 1199, (1962).

[10] R. Balian, Nuevo Cimento 57, 183, (1968).

[11] J.-L. Pichard and P. A. Mello, J. Phys. I, 1, 493, (1991).

[12] R. A. Jalabert, J.-L. Pichard and C. W. J. Beenakker, Europhys. Lett, 27, 255, (1994); H. U. Baranger and P. A. Mello, Phys. Rev. Lett. 73, 142, (1994); R. A. Jalabert and J.-L. Pichard, J. Phys. I France 5, 287, (1995).

[13] F. J. Dyson, J. Math. Phys. 13, 90, (1972).

[14] K. A. Muttalib, J.-L. Pichard and A. D. Stone, Phys. Rev. Lett. 59, 2475, (1987).

[15] C. W. J. Beenakker, Phys. Rev. Lett. 49, 2205, (1994).

[16] O. N. Dorokhov, JETP Lett 36, 318, (1982).

[17] P. A. Mello, P. Pereyra and N. Kumar, Ann. Phys. (N.Y.) 181, 290, (1988).

[18] M. E. Gertsenshstein and V. B. Vasil'ev, Theor. Probab. Appl., 4, 391, (1959).

[19] J.-L. Pichard and G. Sarma, J. Phys. C: Solid State Phys. 14, L127, (1981).

[20] J.-L. Pichard, M. Sanquer, K. Slevin and P. Debray, Phys. Rev. Lett. 65, 1812, (1990).

[21] W. Poirier, D. Mailly and M. Sanquer, Phys. Rev. B 59, 10856, (1999).

[22] Y. Khavin, M. E. Gershenson and A. L. Bogdanov, Phys. Rev. B 58, 8009, (1998).

[23] C. W. J. Beenakker and B. Rejaei, Phys. Rev. Lett. 71, 3689, (1993).

[24] D. Serban, F. Lesage and V. Pasquier, Nucl. Phys. B 466, 499, (1996).

[25] H. U. Baranger and P. A. Mello, cond-mat/9812225 and references therein.

[26] K. B. Efetov and A. I. Larkin, Sov. Phys. JETP 58, 444, (1983).

[27] K. M. Frahm, Phys. Rev. Lett. 74, 4706, (1995).

[28] K. M. Frahm and J.-L. Pichard, J. Phys. I France 5, 847, (1995). 